\title{
Avaliação do uso de um sistema de classificação na assistência de enfermagem: relato de experiência
}

\author{
Evaluation of the use of a classification system in nursing care: experience report
}
Evaluación del uso de un sistema de clasificación en el cuidado de enfermería: informe de experiencia

Elisama da Paz Oliveira Lima ${ }^{1 *}$, Thais de Albuquerque Corrêa ${ }^{1}$, Aniely Tavares da Silva ${ }^{1}$, Luciana da Silva Barreto ${ }^{1}$, Renata Paula Pereira da Silva ${ }^{1}$, Clara de Holanda Braga ${ }^{1}$, Laise Risalva Farias Gouveia da Silva ${ }^{1}$, Camila Dias da Silva Barros ${ }^{1}$, Edjane Maria da Silva ${ }^{2}$, Tatiana Cristina Montenegro Ferreira ${ }^{1}$.

\section{RESUMO}

Objetivo: Relatar a experiência dos estudantes de enfermagem frente ao desenvolvimento do Processo de Enfermagem de acordo com a taxonomia CIPE. Os benefícios de sua aplicação consistem na maior clareza na passagem do plantão, assim como ações de enfermagem mais direcionadas e um atendimento diferenciado. O sistema CIPE, além de auxiliar o enfermeiro na aplicação da SAE, fornece uma linguagem padrão e unificada, elaborada com o intuito de respaldar o exercício na tomada de decisão pelos especialistas. Relato da experiência: A experiência foi vivenciada durante um mês no em um hospital escola, onde eram admitidos pacientes que tiveram episódios de Acidente Vascular Encefálico, assim como derrames e problemas neurológicos associados. A aplicação do SAE segundo o CIPE por parte dos enfermeiros possibilitou uma linguagem específica de enfermagem, sendo possível organizar o trabalho profissional quanto ao método e instrumentos. Considerações finais: Uma terminologia em saúde é importante para a assistência de qualidade. No que tange a importância da SAE segundo o CIPE, os estudantes puderam evidenciar a eficácia do método proposto, sendo notório que o aprendizado aplicado além de ter facilitado o entendimento da disciplina, constrói profissionais capacitados a exercerem o papel com autonomia.

Palavras-chave: Assistência de enfermagem, Processo de enfermagem, Classificação Internacional para a Prática de Enfermagem.

\begin{abstract}
Objective: To report the experience of nursing students regarding the development of the Nursing Process according to the CIPE taxonomy. The benefits of its application consist in greater clarity in the shift shift, as well as more targeted nursing actions and differentiated service. The CIPE system, in addition to assisting nurses in the application of SAE, provides a standard and unified language, designed with the aim of supporting the exercise in decision making by specialists. Experience report: The experience was lived during a month in a teaching hospital, where patients who had episodes of stroke were admitted, as well as strokes and associated neurological problems. The application of the SAE according to the CIPE by the nurses enabled a specific nursing language, making it possible to organize professional work regarding the method and instruments. Discussion: Health terminology is important for quality care. Regarding the importance of SAE according to CIPE, students were able to demonstrate the effectiveness of the proposed method, and it was clear that applied learning, in addition to facilitating the understanding of the discipline, builds professionals trained to exercise their role with autonomy.
\end{abstract}

Keywords: Nursing care, Nursing process, International Classification for Nursing Practice.

${ }^{1}$ Faculdade Pernambucana de Saúde (FPS), Recife - PE. *E-mail: elisamadapazoli@gmail.com

${ }^{2}$ Faculdade de Ciências Humanas de Olinda (FACHO), Olinda - PE. 


\section{RESUMEN}

Objetivo: Informar la experiencia de los estudiantes de enfermería en el desarrollo del Proceso de Enfermería según la taxonomía del CIPE. Los beneficios de su aplicación consisten en una mayor claridad en el turno de turno, así como acciones de enfermería más focalizadas y un servicio diferenciado. El sistema CIPE, además de asistir al enfermero en la aplicación del SAE, proporciona un lenguaje estándar y unificado, diseñado con el objetivo de apoyar el ejercicio en la toma de decisiones por parte de los especialistas. Informe de experiencia: La experiencia se vivió durante un mes en un hospital universitario, donde ingresaron pacientes que tuvieron episodios de ictus, así como ictus y problemas neurológicos asociados. La aplicación del SAE según el CIPE por parte de los enfermeros posibilitó un lenguaje enfermero específico, posibilitando la organización del trabajo profesional en cuanto al método e instrumentos. Discusión: La terminología sanitaria es importante para una atención de calidad. En cuanto a la importancia del SAE según el CIPE, los estudiantes pudieron demostrar la efectividad del método propuesto, y quedó claro que el aprendizaje aplicado, además de facilitar la comprensión de la disciplina, construye profesionales capacitados para ejercer su rol con autonomía.

Palabras clave: Atención de enfermería, Proceso de enfermería, Clasificación Internacional para la Práctica de Enfermería.

\section{INTRODUÇÃO}

A Sistematização da Assistência de Enfermagem (SAE) é uma ferramenta privativa do enfermeiro legalizada pelo Conselho Regional de Enfermagem (COFEN) pela Resolução ${ }^{\circ} 272$ de 2002, na intenção de colaborar com a promoção, prevenção, recuperação e reabilitação do estado saúde e doença do indivíduo (MACHADO JPDC, et al., 2019).

De acordo com o COFEN, na Resolução 358/2009, a SAE é um método que descreve a atuação do enfermeiro, instituindo suas atribuições profissionais e tornado possível a execução do Processo de Enfermagem (PE). Segundo ele, o Processo de Enfermagem consiste em cinco etapas interrelacionadas, interdependentes e recorrentes, sendo elas: Histórico de Enfermagem, Diagnóstico de Enfermagem, Planejamento, Implementação e Evolução de Enfermagem (COFEN, 2009; GARCIA TR e DA NÓBREGA MML, 2009).

A primeira etapa, que consiste no Histórico de Enfermagem, o profissional realizará um levantamento de dados do cliente como também o exame físico. Na segunda etapa é realizado o levantamento dos diagnósticos pertinentes ao estado saúde e doença do paciente. Nesta etapa, o enfermeiro tem a obrigação de identificar as necessidades a serem tratadas, assim como os cuidados que serão prestados (COFEN, 2009; DAL SASSO GTM, et al., 2013).

A terceira etapa, que equivale ao planejamento de enfermagem, ocorre a determinação do resultado, intervenções, ou ações que se espera alcançar. A implementação, que se encaixa como a quarta etapa, é a efetivação das ações e intervenções propostas na etapa do planejamento de enfermagem (ALVIM ALS, 2013; COFEN, 2009).

Na quinta etapa, que transcorre a evolução de enfermagem, será avaliada as respostas do cliente em seu processo saúde e doença para comprovar se as ações e intervenções de enfermagem tiveram o resultado esperado, averiguando a necessidade de modificações nas etapas do processo de enfermagem (COFEN, 2009; BARROS ALBL, et al., 2015).

A implantação da SAE é fundamental para a identificação e reconhecimento dos profissionais de enfermagem, visto que ela é ação privativa do enfermeiro, regulamentada pela lei de número 7489 de 25 de Junho de 1986. Sua utilização com base científica permite uma assistência mais individualizada e pontual, trazendo qualidade ao atendimento (FELIX NN, et al. 2009; BITTENCOURT GKGD, et al., 2008).

Os benefícios de sua utilização consistem na maior clareza na passagem do plantão, assim como ações de enfermagem mais direcionadas e um atendimento diferenciado, específico, ativo e produtivo, pois, esse processo proporciona uma grande relação da enfermeira com o paciente, família e equipe (FELIX NN, et al. 2009). 
A SAE tem se mostrado um método eficaz na administração dos cuidados ao paciente, contudo, na maioria das vezes, é ignorada pelo profissional enfermeiro dentro do ambiente hospitalar, que, por inexperiência, acredita que seu uso como instrumento base no processo de enfermagem faz da atividade um processo enfadonho e burocrático, tendo em vista que o processo requer sincronia em todos os passos. Quando a implantação da SAE é feita de maneira organizada e concisa, os resultados são vistos de forma rápida e eficiente, além de oferecer aos enfermeiros segurança e autonomia nos cuidados prestados (MACHADO JPDC, et al., 2019).

O sistema de Classificação Internacional para a prática de enfermagem (CIPE) fornece uma linguagem padrão e unificada, elaborada com o intuito de respaldar o exercício na tomada de decisão pelos especialistas. É uma terminologia padronizada, extensa e complexa que descreve as atribuições do exercício de enfermagem no âmbito mundial. A mesma proporciona a coleta, o armazenamento, e a análise de dados de enfermagem em uma diversidade de cenário (GARCIA TR, et al., 2018; SANTOS RBD e RAMOS KDS, 2012).

A confirmação desta resolução envolveu a apreensão dos profissionais enfermeiros sobre os problemas e adversidades encontrados para citar situações e complicações com que a enfermagem enfrentava em sua rotina de trabalho, em consequência da ausência de um vocabulário padronizado. As dificuldades percebidas pelos profissionais para especificar a colaboração representativa da enfermagem na prevenção, alívio e solução dos impasses contribuiu para a formulação da resolução (GARCIA TR, et al., 2018).

A classificação internacional para a prática de enfermagem permite ao profissional enfermeiro criar diagnósticos de enfermagem, assim como suas intervenções e, também, seus resultados. Isso concede ao profissional elaborar com mais precisão as ações que serão tomadas para aquele determinado paciente voltado as suas disfunções e particularidades (SIMÕES CMAR e SIMÕE JFFL, 2007; OLEGÁRIO WKB, et al., 2015).

Desta forma, o presente estudo tem como objetivo relatar a experiência dos estudantes de enfermagem do $6^{\circ}$ período da Faculdade Pernambucana de Saúde (FPS) frente ao desenvolvimento do Processo de Enfermagem tendo os diagnósticos, intervenções e resultados propostos de acordo com a CIPE.

\section{RELATO DE EXPERIÊNCIA}

O local da vivência transcorreu em um hospital escola do estado de Pernambuco, no setor da Clínica de Retaguarda onde eram admitidos pacientes que tiveram episódios de acidente vascular encefálico (AVE), assim como derrames e problemas neurológicos associados. O período da prática ocorreu de agosto a setembro de 2019. Os alunos eram supervisionados em toda a vivência prática, tendo um preceptor nomeado pela instituição de ensino para dar suporte em todo o período em que estiveram estagiando.

O estágio supervisionado é um momento de extremo crescimento pessoal e profissional do aluno, pois, por meio de atividades vivenciadas, avaliações e reflexões, proporcionam uma maior confiança e segurança ao final da graduação e início do exercício profissional. É evidente que a construção do embasamento científico e prático do aluno não se dá apenas através da teoria aplicada nas faculdades e universidades, mas também ao conhecimento do discente acerca das práticas vividas.

Inicialmente, foi realizado a visita no setor para ser observado seu funcionamento, assim como suas particularidades. A partir da apresentação por toda a equipe, foram criados vínculos com base em respeito e confiança com os profissionais que executavam atividades pertinentes a sua área.

Com o início da prática, foi observado que a sistematização da assistência de enfermagem se dava no início, com a admissão do paciente. Como o setor era uma área em que eram admitidos pacientes que tiverem algum problema neurológico, muitos clientes chegavam ao local inconscientes, e, algumas vezes, desorientados. A admissão se iniciava no Histórico de enfermagem, onde, utilizava-se um instrumento no modelo de questionário que continham os tópicos a serem preenchidos acerca da saúde do paciente. A entrevista do paciente era realizada através de diálogo, no qual, o profissional ouvia o paciente e também era ouvido pelo mesmo, estabelecendo assim uma relação de confiança. Eram colhidas informações sobre os antecedentes pessoais e hábitos de vida do cliente, assim como, antecedentes familiares, principais queixas e também a história atual da doença. 
Após esta fase, iniciava-se a realização do exame físico, onde era possível observar os sinais vitais, realizar inspeção, palpação, percussão e ausculta. Foi percebido que o profissional de enfermagem a todo momento procurava observar o paciente de maneira holística, considerando todas as informações passadas pelo cliente. É evidente que, neste momento, o enfermeiro precisa de tempo, pois, a partir do exame físico, é feito um levantamento de possíveis diagnósticos significativos. Posto isso, esta fase é extremamente importante e não deve ser ignorada pelo enfermeiro, visto que é necessário paciência e cautela para que o paciente não sofra as consequências de um atendimento mal feito.

Pautados na CIPE, os diagnósticos desenvolvidos seguiam um modelo de questionário pronto, onde o enfermeiro selecionava os mais pertinentes ao atual estado de saúde dos pacientes. Os diagnósticos de maior frequência encontrado na prática foram: "Déficit neurológico", "Déficit cardíaco", "Padrão de eliminação urinária", "Marcha prejudicada", "Integridade tissular prejudicada" e "Déficit do autocuidado". Quanto às intervenções mais recorrentes no setor, estavam presentes: "Registrar alterações de nível de consciência", "Aferir pressão arterial", "Incentivar a deambulação com auxílio", "Registrar fácies de dor" e "Auxiliar no banho de aspersão".

Após o diagnóstico, era refletido o planejamento de enfermagem, no qual consistia em organizar as ações com base nos diagnósticos propostos. Dessa forma, o enfermeiro separava a equipe técnica de enfermagem por distribuições de leitos. O técnico responsável pelo cliente recebia as instruções necessárias para atendêlo, obviamente, sendo monitorado em todo o momento pelo profissional enfermeiro.

Na implementação, o enfermeiro prestava a assistência necessária ao cliente, sendo auxiliado pelo técnico de enfermagem em atividades pertinentes a sua classe. Nas atividades que eram privativas do enfermeiro, as ações eram separadas por ordem, em que, o paciente mais crítico estava sempre em primeiro lugar.

A evolução de enfermagem, que corresponde à quinta etapa do processo de enfermagem, os enfermeiros, ao abrir o plantão, faziam a evolução do paciente, e, ao mesmo tempo, era avaliado o plano de cuidado proposto para o mesmo, observando se houve eficácia e se o paciente estaria ou não evoluindo.

\section{DISCUSSÃO}

Através da literatura buscou-se integrar a Sistematização da Assistência de Enfermagem (SAE) com base nos diagnósticos propostos pela Classificação Internacional para a Pratica de Enfermagem (CIPE). É válido ressaltar que este assunto é de extrema importância para a vida do aluno enquanto acadêmico de enfermagem e ainda mais como futuro profissional enfermeiro.

A SAE deve fazer parte da rotina do enfermeiro, visto que a mesma promove, quando aplicada, inúmeros benefícios para o cuidado empregado, bem como o avanço em bases de dados científicas. A importância conferida à SAE pelo COFEN (órgão este que regulamenta a atividade do profissional enfermeiro) é bem mais extensa do que o PE, pois ela se atenta a parte da gestão do cuidado, viabilizando a competência e êxito do exercício profissional e favorecendo a tomada de decisão. Alguns fatores influenciam na implantação da SAE, tais como: necessidade de apoio da gerência administrativa responsável pela instituição, apoio Inter e entre classes profissionais, recursos físicos, humanos e materiais adequados, capacitação profissional, planejamento da divisão do tempo de trabalho entre atividades administrativas e assistenciais, e desinteresse e despreparo de alguns profissionais.

O Processo de Enfermagem (PE) possibilita ao profissional enfermeiro uma maior autonomia, como também permite que o mesmo participe de todo processo de construção do processo saúde e doença do cliente. Além disso, também permite ao enfermeiro o contínuo exercício do raciocínio crítico, que é de fundamental importância para prestar uma melhor assistência (BITTENCOURT GKGD, et al., 2008).

A aplicação da CIPE viabilizou o PE, pois, através dele, é possível a identificação do diagnóstico e intervenção de enfermagem. A utilização do CIPE no desempenho ocupacional demonstra uma assistência mais capacitada de forma que favorece uma credibilidade maior no desempenho da enfermagem, e, a partir disso, as atividades quando implementadas ganham maior clareza. O CIPE proporciona um cuidar holístico, como também permite o atendimento ao paciente de uma forma mais individualizada, centrando em suas individualidades (BITTENCOURT GKGD, et al., 2008). 
As limitações deste estudo se devem ao fato de os profissionais não terem domínio de associar a teoria da sistematização da assistência de enfermagem (SAE) com a prática, por considerar o método enfadonho, burocrático e que requer do profissional enfermeiro uma maior dedicação de tempo para este cenário. Desta forma, foi possível esclarecer aos estudantes em prática que este método é extremamente necessário e, quando aplicado, respalda as ações dos profissionais, como também promove a promoção, prevenção, recuperação e reabilitação do estado saúde e doença do indivíduo.

Desta maneira, os alunos foram conduzidos durante a vivência prática, estando aptos a associar a teoria com a prática vivida no setor, de forma que os mesmos também se sentiram mais abertos a utilizar a CIPE para elaborar os diagnósticos.

Uma terminologia em saúde é importante para a assistência de qualidade, pois, é capaz de promover uma estrutura para as avaliações clinicamente centradas no paciente, além de conseguir observar atividades de vigilância utilizadas para prevenir complicações, como também confere maior confiabilidade nas ações prestadas pela enfermagem. A SAE possibilita ao profissional enfermeiro mais autonomia nas tomadas de decisões e permite que o mesmo participe de todo o processo saúde e doença do indivíduo, conferindo assim uma assistência mais individualizada. No que tange a importância da SAE segundo o CIPE, os estudantes puderam evidenciar a eficácia do método proposto tanto em sala de aula como diretamente na prática, sendo possível mudar a perspectiva em relação a SAE. Portanto, foi notório que o aprendizado aplicado além de ter facilitado o entendimento da disciplina, constrói profissionais capacitados a exercerem o papel com autonomia.

\section{REFERÊNCIAS}

1. ALVIM ALS. O processo de enfermagem e suas cinco etapas. Enfermagem em foco, 2013; 4(2).

2. BARROS ALBL, et al. Processo de enfermagem: guia para a prática. São Paulo: COREN-SP; 2015.

3. BITTENCOURT GKGD, et al. Assistência de enfermagem a paciente com lúpus eritematoso sistêmico utilizando a CIPE. Revista Gaúcha de Enfermagem, 2008; 29(1): 26.

4. DAL SASSO GTM, et al. Processo de enfermagem informatizado: metodologia para associação da avaliação clínica, diagnósticos, intervenções e resultados. Revista da Escola de Enfermagem da USP, 2013; 47(1): 242-249.

5. COFEN. Conselho Federal de Enfermagem. Dispõe sobre a Sistematização da Assistência de Enfermagem e a implementação do Processo de Enfermagem em ambientes, públicos ou privados, em que ocorre o cuidado profissional de Enfermagem, e dá outras providências. Resolução no358, de, 15, 2009. Disponível em: http://www.abennacional.org.br/site/wp-content/uploads/2019/01/resolucao_358-2009.pdf. Acessado em 15 de julho de 2020.

6. FELIX NN, et al. Desafios encontrados na realização da Sistematização da Assistência de Enfermagem (SAE) em unidade de pronto atendimento. ArqCiênc Saúde, 2009; 16(4): 155-60.

7. GARCIA TR, et al. Classificação internacional para a prática de enfermagem CIPE®: versão 2017. Artmed Editora, 2018.

8. GARCIA TR, DA NÓBREGA MML. Processo de enfermagem: da teoria à prática assistencial e de pesquisa. Escola Anna Nery Revista de Enfermagem, 2009; 13(1): 188-193.

9. DE GUTIÉRREZ MGR; MORAIS SCRV. Sistematização da Assistência de Enfermagem e a formação da identidade profissional. Revista Brasileira de Enfermagem, 2017; 70 (2): 455-460.

10. MACHADO JPDC, et al. Percepção de enfermeiros de unidades de internação clínica sobre a Sistematização da Assistência de Enfermagem. Nursing (São Paulo), 2019; 3220-3225.

11. OLEGÁRIO WKB, et al. Validação de Diagnósticos de Enfermagem da CIPE® para assistência às mulheres no período pós-parto. Revista Eletrônica de Enfermagem, 2015; 17(3).

12. SANTOS RBD, RAMOS KDS. Sistematização da assistência de enfermagem em Centro Obstétrico. Revista Brasileira de Enfermagem, 2012; 65(1): 13-18.

13. SILVA JUNIOR MGD, et al. Software para Sistematização da Assistência de Enfermagem em unidade de internação hospitalar. Revista Brasileira de Enfermagem, 2018; 71(5): 2425-2431.

14. SIMÕES CMAR, SIMÕE JFFL. Avaliação inicial de enfermagem em linguagem CIPE® segundo as necessidades humanas fundamentais. Revista de Enfermagem Referência, 2007; 2(4): 9-23.

15. TAVARES DS, et al. Sistematização da assistência de enfermagem no pré-natal: revisão integrativa. Revista Eletrônica Acervo Saúde, 2019; (31):255-1255. 